\title{
Space Mapping Optimization Algorithms for Engineering Design
}

\author{
Slawomir Koziel ${ }^{1}$, Member, IEEE, John W. Bandler ${ }^{1}$, Fellow, IEEE, and Kaj Madsen ${ }^{2}$ \\ ${ }^{1}$ Department of Electrical and Computer Eng., McMaster University, Hamilton, ON, Canada L8S 4K1 \\ ${ }^{2}$ Informatics and Mathematical Modelling, Technical University of Denmark, DK-2800, Lyngby, Denmark
}

\begin{abstract}
A simple, efficient optimization algorithm based on space mapping $(\mathrm{SM})$ is presented. It utilizes input $\mathrm{SM}$ to reduce the misalignment between the coarse and fine models of the optimized object over a region of interest, and output space mapping (OSM) to ensure matching of response and first-order derivatives between the mapped coarse model and the fine model at the current iteration point. We also consider an enhanced version in which the input $S M$ coefficients are frequency dependent. The performance of our new algorithms is comparable with the recently published SMIS algorithm when applied to a benchmark problem. In comparison with SMIS, the models presented are simple and have a small number of parameters that need to be extracted. The new algorithm is applied to the optimization of a coupled-line band-pass filter.
\end{abstract}

Index Terms-Computer-aided design (CAD), space mapping (SM), optimization, design automation, engineering optimization.

\section{INTRODUCTION}

Space Mapping (SM) technology is now a recognized engineering optimization paradigm, consisting of a number of efficient optimization approaches [1-5]. Direct optimization of an accurate but computationally expensive high fidelity or "fine" model of interest is replaced by the iterative optimization and updating of a so-called "coarse" model (less accurate but cheap to evaluate). If the misalignment between the fine and coarse models is not significant, SM-based algorithms typically provide excellent results after only a few evaluations of the fine model.

SM was originally applied to the optimization of microwave devices [1], where fine models are often based on full-wave electromagnetic (EM) simulators, whereas coarse models are physically-based circuit models. In this case the fine model evaluation can be time consuming. Saving each such evaluation counts. A review and exposition of advances in SM technology is contained in paper [5].

This work was supported in part by the Natural Sciences and Engineering Research Council of Canada under Grant OGP0007239 and Grant STGP269760, and by Bandler Corporation.

S. Koziel is with the Simulation Optimization Systems Research Laboratory, Department of Electrical and Computer Engineering, McMaster University, Hamilton, ON, Canada L8S 4K1.

J.W. Bandler is with the Simulation Optimization Systems Research Laboratory, Department of Electrical and Computer Engineering, McMaster University, Hamilton, ON, Canada L8S 4K1 and also with Bandler Corporation, Dundas, ON, Canada L9H 5E7.

K. Madsen is with Informatics and Mathematical Modelling, Technical University of Denmark, DK-2800, Lyngby, Denmark.
Recent efforts focus on efficient optimization algorithms that use different SM techniques such as implicit space mapping (ISM) [3] and output space mapping (OSM) [4]. SMIS [6] utilizes surrogate models based on SM and OSM and has proved successful for difficult optimization problems. Its performance is obtained, however, at the expense of high complexity of the underlying surrogate model.

This paper describes two simple algorithms that enjoy comparable performance to SMIS. Both exploit surrogate models based on the OSM concept that forces exact matching of responses and Jacobians between surrogate and fine model.

\section{SuRRogate Optimization BASEd ON SPACE MAPPING}

The optimization problem can be stated as follows. Let $R_{f}: X_{f} \rightarrow R^{m}$ denote the response vector of the fine model of a given object, where $X_{\subseteq} \subseteq R^{n}$. In the microwave area [1], [5], components of $\boldsymbol{R}_{\boldsymbol{f}}$ may be evaluations of the fine model (e.g., transfer function) at $m$ different frequency points.

Our goal is to solve

$$
\boldsymbol{x}_{f}^{*}=\arg \min _{\boldsymbol{x} \in X_{f}} U\left(\boldsymbol{R}_{f}(\boldsymbol{x})\right)
$$

where $U$ is a given objective function. We assume that solving problem (1) by means of direct optimization is impractical. Instead, we exploit inexpensive surrogates. We are interested in a general optimization algorithm that generates a sequence of points $\boldsymbol{x}^{(i)} \in X_{f}, i=1,2, \ldots$, and a family of surrogate models $R_{s}^{(i)}: X_{s}^{(i)} \rightarrow R^{m}, i=0,1, \ldots$, so that

$$
\boldsymbol{x}^{(i+1)}=\arg \min _{\boldsymbol{x} \in X_{i}^{(i)} \cap X_{f}} U\left(\boldsymbol{R}_{s}^{(i)}(\boldsymbol{x})\right)
$$

and $R_{s}^{(i+1)}$ is constructed using suitable matching conditions with the fine model at previous points $\boldsymbol{x}^{(k)}, k=1, \ldots, i$.

Space mapping assumes an underlying coarse model that describes the same object as the fine model; is less accurate but, at the same time, much faster to evaluate than the fine model. Let $\boldsymbol{R}_{c}: X_{c} \rightarrow R^{m}$ denote the response vectors of the coarse model, where $X_{f} \subseteq R^{n}$. By $\boldsymbol{x}_{c}^{*}$ we shall denote the optimal solution of the coarse model, i.e.,

$$
\boldsymbol{x}_{\boldsymbol{c}}^{*}=\arg \min _{\boldsymbol{x} \in X_{c}} U\left(\boldsymbol{R}_{c}(\boldsymbol{x})\right)
$$

In the SM framework, a family of surrogate models is constructed from the coarse model in such a way that each $\boldsymbol{R}_{s}^{(i)}$ is a suitable distortion of $\boldsymbol{R}_{c}$, such that given matching conditions are satisfied. In the next sections we introduce two SM-based models that fit this scheme. 


\section{A GSM FRAMEWORK}

\section{A. Proposed Framework}

Our proposed generalized space mapping (GSM) framework consists of the family of surrogate models $\boldsymbol{R}_{s}^{(i)}$ defined as

$$
\boldsymbol{R}_{s}^{(i)}(\boldsymbol{x})=\boldsymbol{A}^{(i)} \cdot \boldsymbol{R}_{c}\left(\boldsymbol{B}^{(i)} \cdot \boldsymbol{x}+\boldsymbol{c}^{(i)}\right)+\boldsymbol{d}^{(\hat{)})}+E^{(i)} \cdot\left(\boldsymbol{x}-\boldsymbol{x}^{(i)}\right)
$$

where

$$
\begin{gathered}
\left(A^{(i)}, B^{(i)}, c^{(i)}\right)=\arg \min _{(A, B, c)} \varepsilon^{(i)}(A, B, c) \\
\boldsymbol{d}^{(i)}=R_{f}\left(\boldsymbol{x}^{(i)}\right)-A^{(i)} \cdot \boldsymbol{R}_{c}\left(\boldsymbol{B}^{(i)} \cdot \boldsymbol{x}^{(i)}+\boldsymbol{c}^{(i)}\right) \\
\boldsymbol{E}^{(i)}=J_{R_{f}}\left(\boldsymbol{x}^{(i)}\right)-\boldsymbol{A}^{(i)} \cdot \boldsymbol{J}_{\boldsymbol{R}_{c}}\left(\boldsymbol{B}^{(i)} \cdot \boldsymbol{x}^{(i)}+\boldsymbol{c}^{(i)}\right) \cdot \boldsymbol{B}^{(i)}
\end{gathered}
$$

Matrices $A^{(i)}=\operatorname{diag}\left\{a_{1}^{(i)}, \ldots, a_{m}^{(i)}\right\}, B^{(i)} \in M_{D \times n}$ and $c^{(i)} \in M_{D \times 1}$ are obtained using parameter extraction applied to the matching condition $\varepsilon^{(\hat{i})}$. Matrices $\boldsymbol{d}^{(\hat{)}} \in M_{m \times 1}$ and $\boldsymbol{E}^{(\hat{i})} \in M_{m \times n}$ are calculated using formulas (6) and (7) after having determined $A^{(i)}, B^{(i)}, c^{(i)}$. If derivative information is not available, matrix $E^{(\lambda)}$ can be estimated, for example, using the Broyden update.

The matching condition $\varepsilon^{(i)}$ determines the surrogate model as much as formula (4) does. We can consider different matching conditions that aim at aligning the fine and surrogate model responses and/or their first-order derivatives. A general form of the matching condition is

$$
\begin{gathered}
\varepsilon^{(i)}(\boldsymbol{A}, \boldsymbol{B}, \boldsymbol{c})=\sum_{k=0}^{i} W_{k}\left\|R_{f}\left(\boldsymbol{x}^{(k)}\right)-\boldsymbol{A} \cdot \boldsymbol{R}_{c}\left(\boldsymbol{B} \cdot \boldsymbol{x}^{(k)}+\boldsymbol{c}\right)\right\|+ \\
+\sum_{k=0}^{i} V_{k}\left\|J_{R_{f}}\left(\boldsymbol{x}^{(k)}\right)-\boldsymbol{A} \cdot \boldsymbol{J}_{\boldsymbol{R}_{c}}\left(\boldsymbol{B} \cdot \boldsymbol{x}^{(k)}+\boldsymbol{c}\right) \cdot \boldsymbol{B}\right\|
\end{gathered}
$$

We assume that coefficients $W_{k}$ and $v_{k}$ are either 0 or 1 (although more general situations are conceivable). Setting $W_{k}=1, k=0, \ldots, i$ and $v_{k}=0, k=0, \ldots, i-1, V_{i}=1$ means that the surrogate tries to match the fine model response at all previous points $x^{(t)}$ (including current point) as well as Jacobian at the current point (Jacobian matching can only be exploited if derivative information is available). We shall use this condition in the subsequent numerical experiments concerning model (4).

Input space mapping determined by matrices $B^{(i)}$ and $c^{(\hat{i})}$ as well as multiplication matrix $A^{(i)}$ can be considered as preconditioning of the coarse model that reduces initial misalignment between the coarse and fine models over a neighborhood of the current point $\left(\boldsymbol{x}^{(\hat{i})}\right)$. Term $\boldsymbol{d}^{(i)}$ ensures perfect matching of responses at $\boldsymbol{x}^{(i)}$, while term $\boldsymbol{E}^{(i)}$ gives perfect matching of first-order derivatives at $\boldsymbol{x}^{(i)}$.

Note that the flexibility of the surrogate model, i.e., the number of model parameters, may affect the uniqueness of the parameter extraction problem as well as the extrapolation properties of the model. If the model is too flexible, it may provide good matching at the points $\boldsymbol{x}^{(k)}$ but provide poor matching elsewhere, and, consequently, slow down (or even prevent) convergence of the SM algorithm. The issue of proper choice of flexibility of the surrogate model is an important problem and needs to be carefully studied.

Note that the number of GSM model parameters is just $n(n+1)+m(n+2)$, and only $n(n+1)+m$ parameters are extracted (the rest are calculated). Moreover, the model ensures perfect matching of the response and Jacobian at the current point $\boldsymbol{x}^{(\hat{)})}$.
Having defined the family of surrogate models we can define the optimization algorithm (which is, in fact, an implementation of the generic surrogate model based optimization algorithm (2)):

Step 1 Set $\boldsymbol{x}^{(0)}=\arg \min _{\boldsymbol{x} \in X_{f} \cap X_{c}} U\left(\boldsymbol{R}_{c}(\boldsymbol{x})\right)$; Set $i=0$;

Step 2 Evaluate $\boldsymbol{R}_{f}\left(\boldsymbol{x}^{(i)}\right)$ and $\boldsymbol{J}_{\boldsymbol{R}_{f}}\left(\boldsymbol{x}^{(i)}\right)$;

Step 3 Obtain $\boldsymbol{R}_{s}^{(i)}$ using (4)-(8);

Step 4 Find $\boldsymbol{x}^{(i+1)}=\arg \min _{\boldsymbol{x} \in X_{s}^{(i)} \cap X_{f}} U\left(\boldsymbol{R}_{s}^{(i)}(\boldsymbol{x})\right)$;

Step 5 If termination condition go to 7 ;

Step 6 Set $i:=i+1$; go to 2;

Step 7 END

In numerical experiments we use a termination condition of the form $\left\|\boldsymbol{x}^{(i+1)}-\boldsymbol{x}^{(i)}\right\|<\delta$, where $\delta$ is a small constant.

\section{B. Comparison with SMIS}

Our GSM framework draws on the strengths of the recently introduced SMIS algorithm (space mapping interpolating surrogates) [6]. SMIS is, however, complex because it uses a separate input mapping for each component of the response vector. As a consequence, the mapped coarse model is only defined on the discrete set of frequency points $\omega_{1}, \ldots, \omega_{m}$ instead of the whole frequency domain. This is not the case for our GSM, which uses only one input mapping. Another advantage of GSM is the number of model parameters to be extracted, $n(n+1)+m$, compared with $m \cdot(n(n+1)+2$ in SMIS, where $m$ is larger than $n$. A large number of parameters may result in lack of uniqueness in the parameter extraction process as well as poor extrapolation properties of the model so that it cannot be effectively used in circuit modeling.

\section{THE FDGSM FRAMEWORK}

Our GSM based surrogate model (4)-(8) is simple. But in some cases the number of its parameters may not be sufficient to ensure proper matching between the fine model and the surrogate. This affects the convergence of the underlying optimization algorithm. If necessary, the number of degrees of freedom should be increased. One possibility is to use frequency-dependent mappings.

FDGSM (Frequency-Dependent General Space Mapping) is an extension of our framework in which the input space mapping parameters (matrices $B$ and $\boldsymbol{c}$ ) are made frequency-dependent. Let $R_{f}: X_{f} \times \Omega \rightarrow R$ and $R_{c}: X_{c} \times \Omega \rightarrow R$ denote generic fine and coarse models, where $\Omega$ is the frequency domain. Then, $\boldsymbol{R}_{\boldsymbol{f}}(\boldsymbol{x})=\left(R_{f, 1}(\boldsymbol{x}), \ldots, R_{f, m}(\boldsymbol{x})\right)$, and $\boldsymbol{R}_{c}(\boldsymbol{x})=\left(R_{c .1}(\boldsymbol{x}), \ldots, R_{c . m}(\boldsymbol{x})\right), \quad$ with $\quad R_{f, i}(\boldsymbol{x})=R_{f}\left(\boldsymbol{x}, \omega_{i}\right)$, $R_{c, i}(\boldsymbol{x})=R_{c}\left(\boldsymbol{x}, \omega_{i j}\right), i=1, \ldots, m$. A formal definition of the model is

$$
\overline{\boldsymbol{R}}_{s}^{(i)}(\boldsymbol{x})=A^{(i)} \cdot \overline{\boldsymbol{R}}_{c}\left(\boldsymbol{x}, \boldsymbol{B}^{(i)}, c^{(i)}\right)+\boldsymbol{d}^{(i)}+E^{(i)} \cdot\left(\boldsymbol{x}-\boldsymbol{x}^{(j)}\right)
$$

where

$$
\begin{aligned}
\overline{\boldsymbol{R}}_{c}\left(\boldsymbol{x}, \boldsymbol{B}^{(i)}, c^{(i)}\right)= & \left(R_{c}\left(\boldsymbol{B}^{(i)}\left(\omega_{1}\right) \cdot \boldsymbol{x}+\boldsymbol{c}^{(i)}\left(\omega_{1}\right), \omega_{1}\right), \ldots,\right. \\
& \left.R_{c}\left(\boldsymbol{B}^{(i)}\left(\omega_{m}\right) \cdot \boldsymbol{x}+\boldsymbol{c}^{(i)}\left(\omega_{m}\right), \omega_{m}\right)\right)
\end{aligned}
$$




$$
\begin{gathered}
\boldsymbol{B}^{(i)}(\omega)=\boldsymbol{B}_{0}^{(i)}+\boldsymbol{B}_{1}^{(i)} \cdot \omega, \quad \boldsymbol{c}^{(i)}(\omega)=\boldsymbol{c}_{0}^{(i)}+\boldsymbol{c}_{1}^{(i)} \cdot \omega \\
\left(\boldsymbol{A}^{(i)}, \boldsymbol{B}^{(i)}, \boldsymbol{c}^{(i)}\right)=\arg \min _{\left(\boldsymbol{A}_{B}, \boldsymbol{c}, \varepsilon^{(i)}\right.}(A, \boldsymbol{B}, \boldsymbol{c}) \\
\boldsymbol{d}^{(i)}=\boldsymbol{R}_{f}\left(\boldsymbol{x}^{(i)}\right)-\boldsymbol{A}^{(i)} \cdot \overline{\boldsymbol{R}}_{c}\left(\boldsymbol{x}^{(i)}, \boldsymbol{B}^{(i)}, \boldsymbol{c}^{(i)}\right) \\
\boldsymbol{E}^{(i)}=J_{\boldsymbol{R}_{f}}\left(\boldsymbol{x}^{(i)}\right)-J_{\bar{R}_{s}^{(i)}}\left(\boldsymbol{x}^{(i)}\right)
\end{gathered}
$$

Matrices $\quad A^{(i)}=\operatorname{diag}\left\{a_{1}^{(i)}, \ldots, a_{m}^{(i)}\right\}, \quad \boldsymbol{B}_{0}^{(i)}, \boldsymbol{B}_{1}^{(i)} \in M_{n \times n}$, and $c_{0}^{(i)}, c_{1}^{(i)} \in M_{n \times 1}$ are obtained using parameter extraction applied to the matching condition $\varepsilon^{(i)}$. Matrices $\boldsymbol{d}^{(i)} \in M_{m \times 1}$ and $E^{(i)} \in M_{m \times n}$ are calculated using formulas (13) and (14) after having determined $\boldsymbol{A}^{(i)}, \boldsymbol{B}^{(i)}, \boldsymbol{c}^{(i)}$. Matching condition $\varepsilon^{(i)}$ takes a similar form as in the previous case, namely,

$$
\begin{aligned}
\varepsilon^{(i)}(A, B, c) & =\sum_{k=0}^{i} W_{k}\left\|R_{f}\left(\boldsymbol{x}^{(k)}\right)-A \cdot \bar{R}_{c}\left(\boldsymbol{x}^{(k)}, B, c\right)\right\|+ \\
& +\sum_{k=0}^{i} V_{k}\left\|J_{R_{f}}\left(\boldsymbol{x}^{(k)}\right)-J_{\boldsymbol{R}_{s}^{(j)}}\left(\boldsymbol{x}^{(k)}\right)\right\|
\end{aligned}
$$

In numerical experiments we assumed $w_{k}=1, k=0, \ldots, i$ and $v_{k}=0, k=0, \ldots, i$ (matching of responses at all points $\boldsymbol{x}^{(k)}$ ). The Jacobian $J_{\bar{R}_{s}^{(t)}}\left(\boldsymbol{x}^{(k)}\right)$ is calculated as

$$
J_{\bar{R}_{s}^{(n)}}\left(\boldsymbol{x}^{(k)}\right)=\boldsymbol{A}^{(i)}\left[\begin{array}{c}
\nabla_{\boldsymbol{x}} R_{c}\left(\boldsymbol{B}^{(i)}\left(\omega_{1}\right) \cdot \boldsymbol{x}^{(k)}+\boldsymbol{c}^{(j)}\left(\omega_{1}\right), \omega_{1}\right) \cdot \boldsymbol{B}^{(i)}\left(\omega_{1}\right) \\
\vdots \\
\nabla_{\boldsymbol{x}} R_{c}\left(\boldsymbol{B}^{(j)}\left(\omega_{m}\right) \cdot \boldsymbol{x}^{(k)}+\boldsymbol{c}^{(i)}\left(\omega_{m}\right), \omega_{m}\right) \cdot \boldsymbol{B}^{(j)}\left(\omega_{m}\right)
\end{array}\right]
$$

Note that the number of model parameters in FDGSM is $2 n(n+1)+m(n+2)$, with $2 n(n+1)+m$ parameters to be extracted. As in GSM, the model ensures perfect matching of the response and Jacobian at the current point $\boldsymbol{x}^{(i)}$. The optimization algorithm is the same as in Section III.

\section{EXAMPLES}

We apply the proposed GSM and FDGSM algorithms to the seven-section capacitively-loaded impedance transformer [7]. Here $U=\max _{1 \leq i \leq m}\left|S_{11, i}\right| \quad\left(S_{11, i}\right.$ is the input reflection coefficient at frequency $\omega_{i j}$ ). We consider a "coarse" model as an ideal seven-section transmission line (TL), whereas the "fine" model is a capacitively-loaded TL with capacitors $C_{1 \ldots 8}=0.025 \mathrm{pF}$. The models are shown in Fig. 1. Design parameters are normalized lengths $\boldsymbol{x}=\left[\begin{array}{lllllll}L_{1} & L_{2} & L_{3} & L_{4} & L_{5} & L_{6} & L_{7}\end{array}\right]^{T}$, with respect to the quarter-wave length $L_{q}$ at the center frequency $4.35 \mathrm{GHz}$. Design specifications are $\left|S_{11}\right| \leq 0.07$ for $1 \mathrm{GHz} \leq \omega \leq 7.7 \mathrm{GHz}$ with 68 points per frequency sweep (for the sake of comparison, the number of frequency points has been chosen to be the same as in the SMIS algorithm [6]).

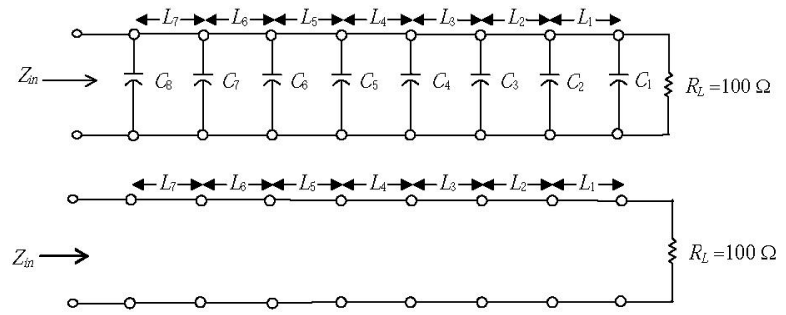

Fig. 1. Seven-section capacitively-loaded impedance transformer: "fine" model (upper graph) and "coarse" model (lower graph).
We solve the PE problem using the nonlinear least squares Levenberg-Marquardt algorithm available in the Matlab Optimization Toolbox [8].

The fine model response at the optimal coarse model solution is shown in Fig. 2. Fig. 3 shows the difference at iteration $i$ between the fine model objective function $U^{(i)}$ and the fine model objective function at the fine model minimax solution $U^{*}$. The difference at iteration $i$ between the fine model design $\boldsymbol{x}^{(i)}$ and the fine model design at the fine model minimax solution $\boldsymbol{x}^{*}$ is shown in Fig. 4.

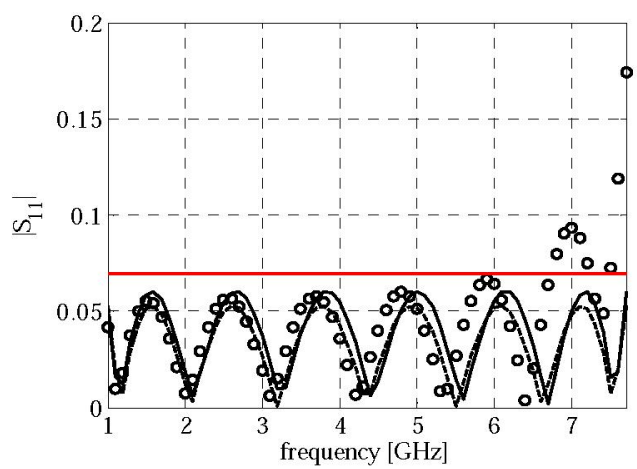

Fig. 2. Seven-section capacitively-loaded impedance transformer: optimal coarse model response (dashed line), the optimal minimax fine model response (solid line), and the fine model response at the initial solution, i.e., at the optimal coarse model solution (circles).

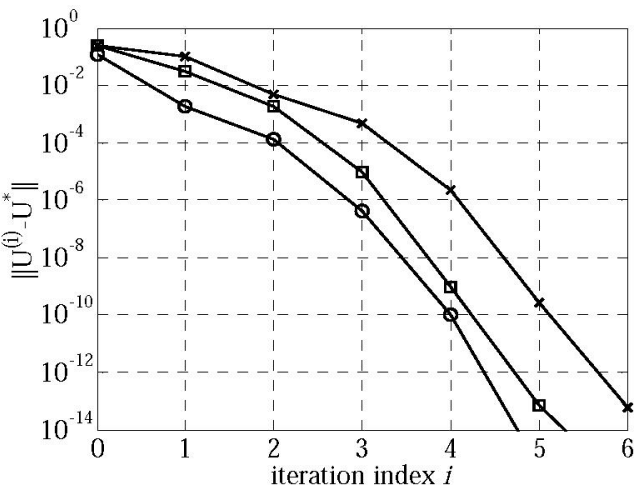

Fig. 3. The difference at iteration $i$ between the fine model objective function $U^{(i)}$ and the fine model objective function at the fine model minimax solution $U^{*}: \operatorname{GSM}(\times)$, FDGSM ( $\square$ ), and SMIS [9] (o).

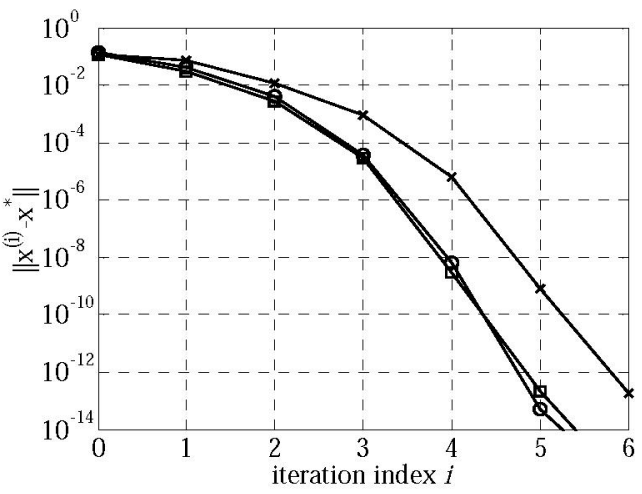

Fig. 4 . The difference at iteration $i$ between the fine model design $\boldsymbol{x}^{(i)}$ and the fine model design at the fine model minimax solution $\boldsymbol{x}^{*}$ : GSM $(\times)$, FDGSM (), and SMIS [9] (o). 
The results indicate that the performance of GSM is only slightly worse than SMIS, whereas FDGSM performs virtually as well as SMIS. It should be emphasized that the number of model parameters for this benchmark problem is much smaller for GSM (668 but only 124 to extract) and FDGSM (724; 180 to be extracted) than for SMIS (3944; 3876 to be extracted).

The GSM framework was also applied to the optimization of the coupled-line band-pass filter [9] shown in Fig. 5. The design parameters are $\boldsymbol{x}=\left[\begin{array}{llll}X_{1} & X_{2} & X_{3} & X_{4}\end{array}\right]^{T}$. The fine model is simulated in FEKO [10], the coarse model is the circuit model implemented in Agilent ADS [11] (Fig. 6). The design specifications are $\left|S_{21}\right| \leq-20 \mathrm{~dB}$ for $6 \mathrm{GHz} \leq f \leq 7.2 \mathrm{GHz}$, $\left|S_{21}\right| \geq-3 \mathrm{~dB} \quad$ for $7.8 \mathrm{GHz} \leq f \leq 8.2 \mathrm{GHz}$, and $\left|S_{21}\right| \leq-20 \mathrm{~dB}$ for $8.8 \mathrm{GHz} \leq f \leq 10 \mathrm{GHz}$.

The initial design is coarse model optimal solution $\boldsymbol{x}^{(0)}=\left[\begin{array}{llll}1.096 & 6.159 & 1.798 & 7.092\end{array}\right]^{T} \mathrm{~mm}$. The fine model response at $\boldsymbol{x}^{(0)}$ as well as the response at the solution obtained using

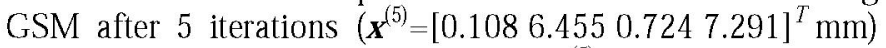
are shown in Fig. 7 . The response at $\boldsymbol{x}^{(5)}$ satisfies the design specifications. In this case we constrained matrix $A$ to be $\operatorname{diag}\{1, \ldots, 1\}$ to simplify the algorithm. The Jacobian of the fine/coarse model was estimated using finite differences. The convergence graph is shown in Fig. 8.

\section{CONCLUSIONS}

New and efficient optimization algorithms are presented that use "traditional" input-space-mapping-based preconditioning of the coarse model and output-spacemapping external terms ensuring perfect matching of the response and first-order derivative between the surrogate and the fine model. We consider the basic model (GSM) and an enhanced version (FDGSM) that demonstrates how the number of degrees of freedom of the SM based models can be increased in a consistent way using frequency-dependent mappings. Performance of the algorithm is illustrated through a seven-section capacitively-loaded impedance transformer and a coupled-line band-pass filter.

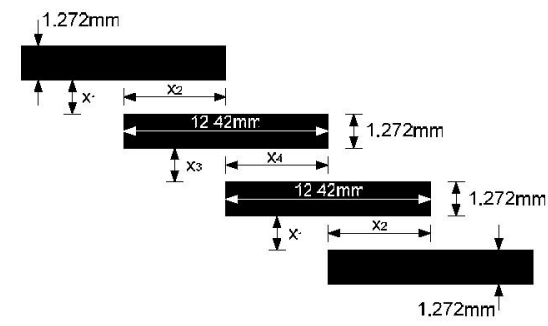

Fig. 5. Geometry of the band-pass filter [9].

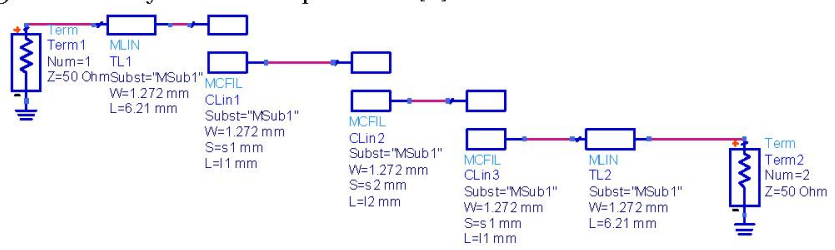

Fig. 6. Coarse model of band-pass filter (ADS).

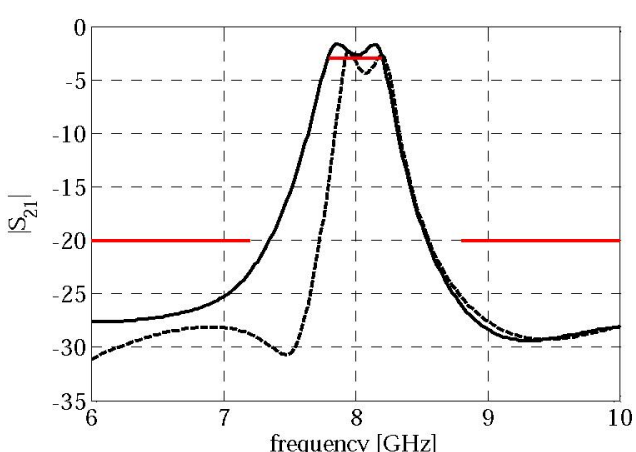

Fig. 7. Initial (dashed line) and optimized (solid line) $\left|S_{21}\right|$ versus frequency for the band-pass filter.

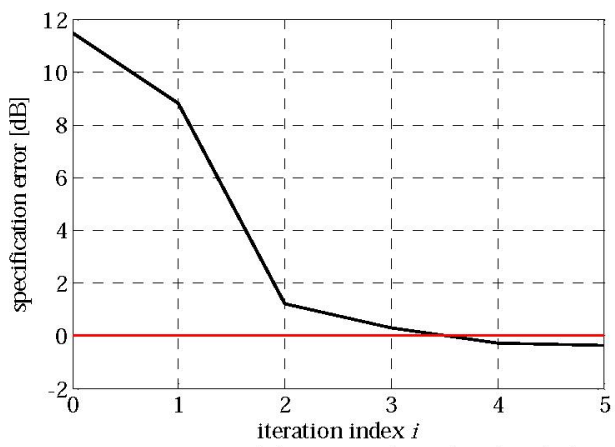

Fig. 8. Convergence properties of the GSM algorithm for the band-pass filter.

\section{REFERENCES}

[1] J.W. Bandler, R.M. Biernacki, S.H. Chen, P.A. Grobelny and R.H Hemmers, "Space mapping technique for electromagnetic optimization," IEEE Trans. Microwave Theory Tech., vol. 2, pp. 536-544, Dec. 1994.

[2] J.W. Bandler, R.M. Biernacki, S.H. Chen, R.H. Hemmers and K. Madsen, "Electromagnetic optimization exploiting aggressive space mapping," IEEE Trans. Microwave Theory Tech., vol. 43, pp. 28742882, Dec. 1995.

[3] J.W. Bandler, Q.S. Cheng, N.K. Nikolova and M.A. Ismail, "Implicit space mapping optimization exploiting preassigned parameters," IEEE Trans. Microwave Theory Tech., vol. 52, pp. 378-385, Jan. 2004.

[4] J.W. Bandler, Q.S. Cheng, D.H. Gebre-Mariam, K. Madsen, F. Pedersen and J. Søndergaard, "EM-based surrogate modeling and design exploiting implicit, frequency and output space mappings," IEEE MTT-S IMS Digest, Philadelphia, PA, 2003, pp. 1003-1006.

[5] J.W. Bandler, Q.S. Cheng, S.A. Dakroury, A.S. Mohamed, M.H. Bakr, K. Madsen and J. Sondergaard, "Space mapping: the state of the art," IEEE Trans. Microwave Theory Tech, vol. 52, pp. 337-361, Jan. 2004.

[6] J.W. Bandler, D.M. Hailu, K.Madsen and F. Pedersen, "A space mapping interpolating surrogate algorithm for highly optimized EM-based design of microwave devices," IEEE Trans. Microwave Theory Tech., vol.52, pp. 2593-2600, Nov. 2004.

[7] M.H. Bakr, J.W. Bandler, K. Madsen and J. Søndergaard, "An introduction to the space mapping technique," Optimization and Engineering, vol. 2, 2001, pp. 369-384.

[8] Matlab ${ }^{\mathrm{TM}}$, Version 7.0, The MathWorks, Inc., 3 Apple Hill Drive, Natick MA 01760-2098, 2002.

[9] E.A. Soliman, M.H. Bakr and N.K. Nikolowa, "Accelerated gradient-based optimization of planar circuits," IEEE Trans. Antennas and Propagation, vol. 53, 2005, pp. 880-883.

[10] FEKO ${ }^{\circledast}$ User's Manual, Suite 4.2, June 2004, EM Software \& SystemsS.A. (Pty) Ltd, 32 Techno Lane, Technopark, Stellenbosch, 7600, South Africa, http://www.feko.info.

[11] Agilent ADS, Version 2003C, Agilent Technologies, 1400 Fountaingrove Parkway, Santa Rosa, CA 95403-1799, 2003. 